Supporting information for:

\title{
Disordered two-dimensional self-organization of ethyl pyruvate molecules on the $\operatorname{Pt}(111)$ surface
}

C. Witt, M. C. Schmidt, C. Schröder, S. Schauermann, and B. Hartke*

Institute for Physical Chemistry,

Christian-Albrechts-University,

Olshausenstr. 40, 24098 Kiel, GERMANY

*e-mail: hartke@pctc.uni-kiel.de 


\section{Convergence with respect to number of layers}

To illustrate how properties of ethyl pyruvate (EP) oligomers on $\mathrm{Pt}(111)$ change with the number of Pt layers, we show two characteristic examples here. In both cases, we have taken a randomly selected EP dimer from our global optimization runs, locally optimal but not the globally best one, to be more representative for the course of the optimization, not only its end.

Fig. S1 shows partial charges on the four most strongly charged atoms in one of these two EP molecules, namely the three oxygen atoms and the ester carbon atom, as a function of the number of $\mathrm{Pt}$ layers, from 1 through 8. Note that these were single-point calculations, i.e., without changing or optimizing any atom positions (which could induce partial charge changes). These partial charges range from +0.1456 for the ester C-atom to -0.4835 for the ester-carbonyl O-atom. Hence, to capture all these data in one plot and to make partial charge changes discernible, not the partial charges themselves are shown but rather their difference to the situation with $8 \mathrm{Pt}$ layers (where these differences are zero by construction). Using just one Pt layer clearly would be insufficient. However, considering the rather small values on the ordinate, partial charges are close to convergence already at just $2 \mathrm{Pt}$ layers and can be considered converged at 4 layers or more.

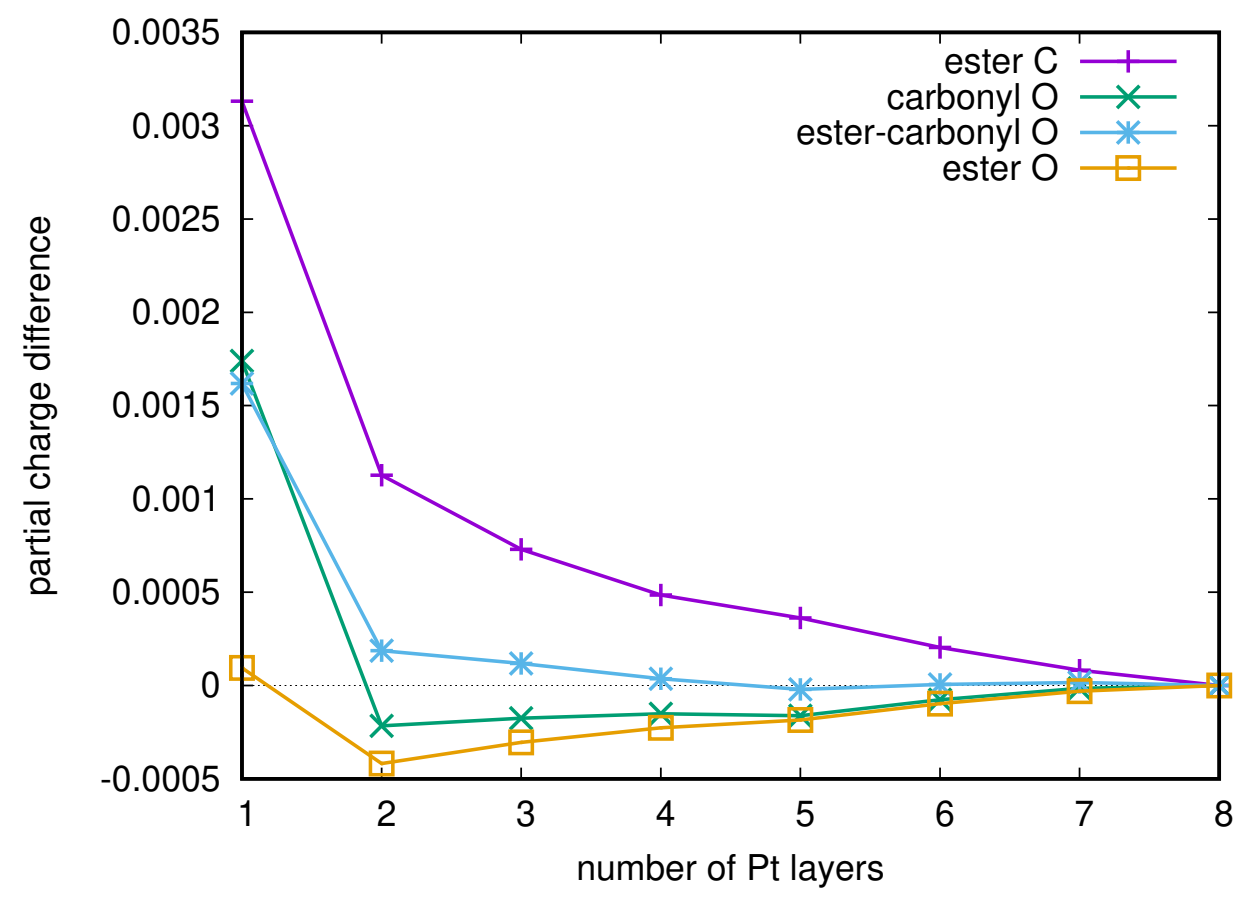

Figure S1: Partial charges on four selected atoms of one EP molecule in an EP dimer on a $\mathrm{Pt}$ slab, for varying number of $\mathrm{Pt}$ layers, shown as charge differences between the situation with 8 layers and other ones with fewer layers, down to just one layer.

For the same EP dimer on $\mathrm{Pt}(111)$, Fig. S2 illustrates how the total surface-binding energy of this EP dimer (i.e., the sum of the binding energies of the two EP molecules) changes when the number of $\mathrm{Pt}$ layers is varied, again between 1 and 8 . Here, using 2 layers clearly is not sufficient, whereas 4 or more layers can be considered converged. Non-monotonic oscillations in the range of 4-8 layers in both Figs. S1 and S2 are likely 
due to the smaller $x y$-extent of the surface slab, which was chosen here to be able to accomodate up to 8 layers in the ХTB program for these test calculations. In our production runs, the $x y$-extent was always larger.

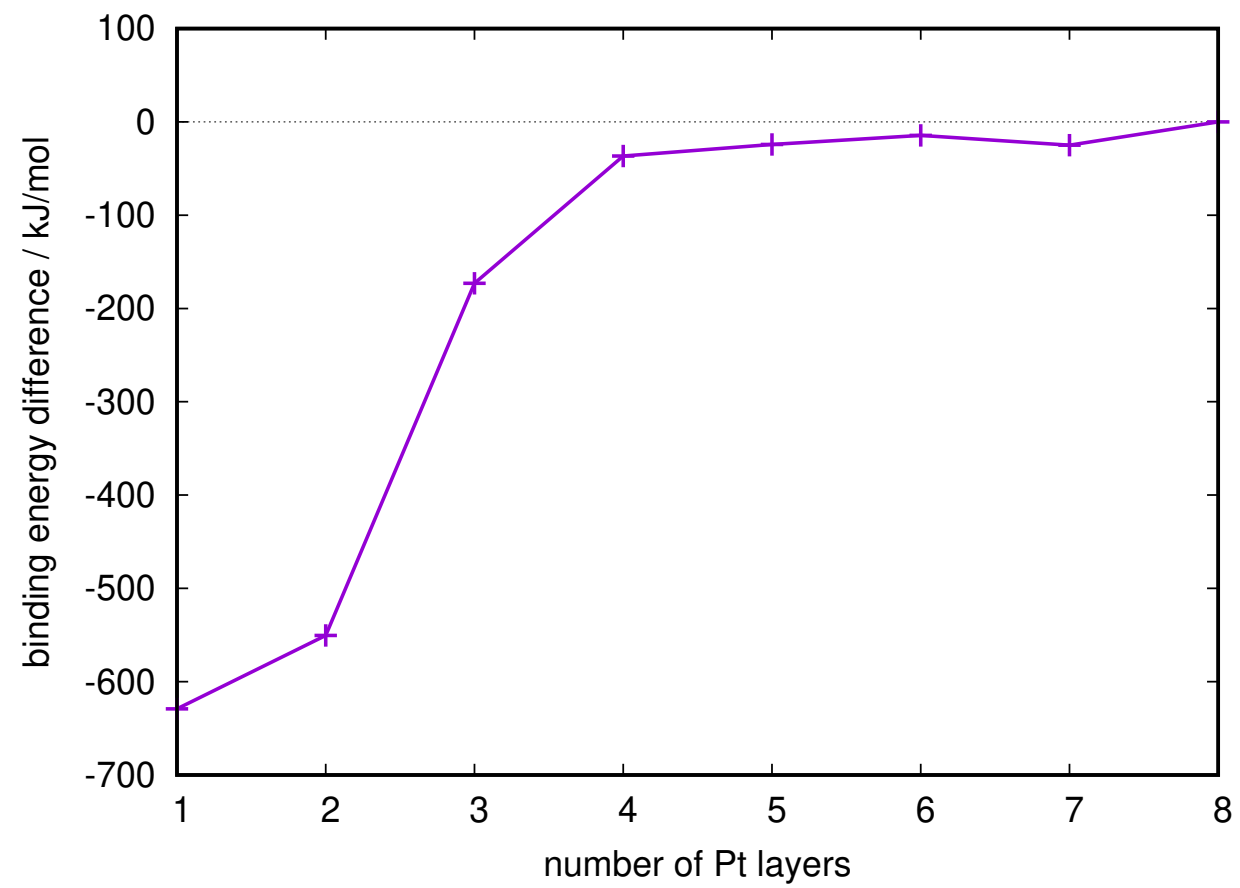

Figure S2: Total binding energy of an EP dimer on a $\mathrm{Pt}$ slab, for varying number of $\mathrm{Pt}$ layers, as in Fig. S1 shown as difference between the 8-layers situation and those with fewer layers.

Note that binding energy differences between different EP oligomer structures on $\mathrm{Pt}(111)$, and to an even larger extent the energy ranking of such isomers, are less sensitive to changes in the number of Pt layers. For our global optimization strategy, essentially only the energy ranking is relevant.

Even in high-end density-functional theory studies of molecular adsorption on surfaces, 6-layer slabs are the frequent choice [1]. in our previous study of triazatriangulene (TATA) molecules on $\mathrm{Au}(111)$, even more extensive convergence tests [2] lead us to use 3-layer slabs, with parameters adapted to mimic 9-layer slabs. However, in that case the molecule-surface interaction was purely van-der-Waals, hence higher accuracy was mandatory. Here we do not have indications that more than 6 layers are needed.

\section{Fixed vs. relaxed Pt-layers}

In contrast to soft coinage metals like gold with its famous herringbone reconstruction [3], $\operatorname{Pt}(111)$ is less prone to surface reconstructions. In fact, in our scanning tunneling microscopy studies of EP@Pt(111), no surface reconstruction upon EP adsorption was detected.

Computational experiments in the present GFN-FF setup support this finding: If we do not keep all Pt layers fixed but constrain the upper layers with harmonic potentials [4] of different force constants, this does change properties like absolute surface-binding energies to some extent, but the binding energy ordering of EP oligomer isomers on 
$\operatorname{Pt}(111)$, which is relevant for our global optimization, is unchanged - in fact, even the binding energy differences remain the same in a semi-quantitative fashion.

This is illustrated in Fig. S3, comparing binding energies of three randomly selected, different EP dimers to that of a fourth one, for three different scenarios: a fully rigid Pt slab, and Pt slabs with relaxed top layers, constrained by harmonic-oscillator force constants of 0.05 and 0.01 hartree/bohr ${ }^{2}$. Again, all of these dimers are locally optimized and of low energy, but not globally optimal, to be more representative.

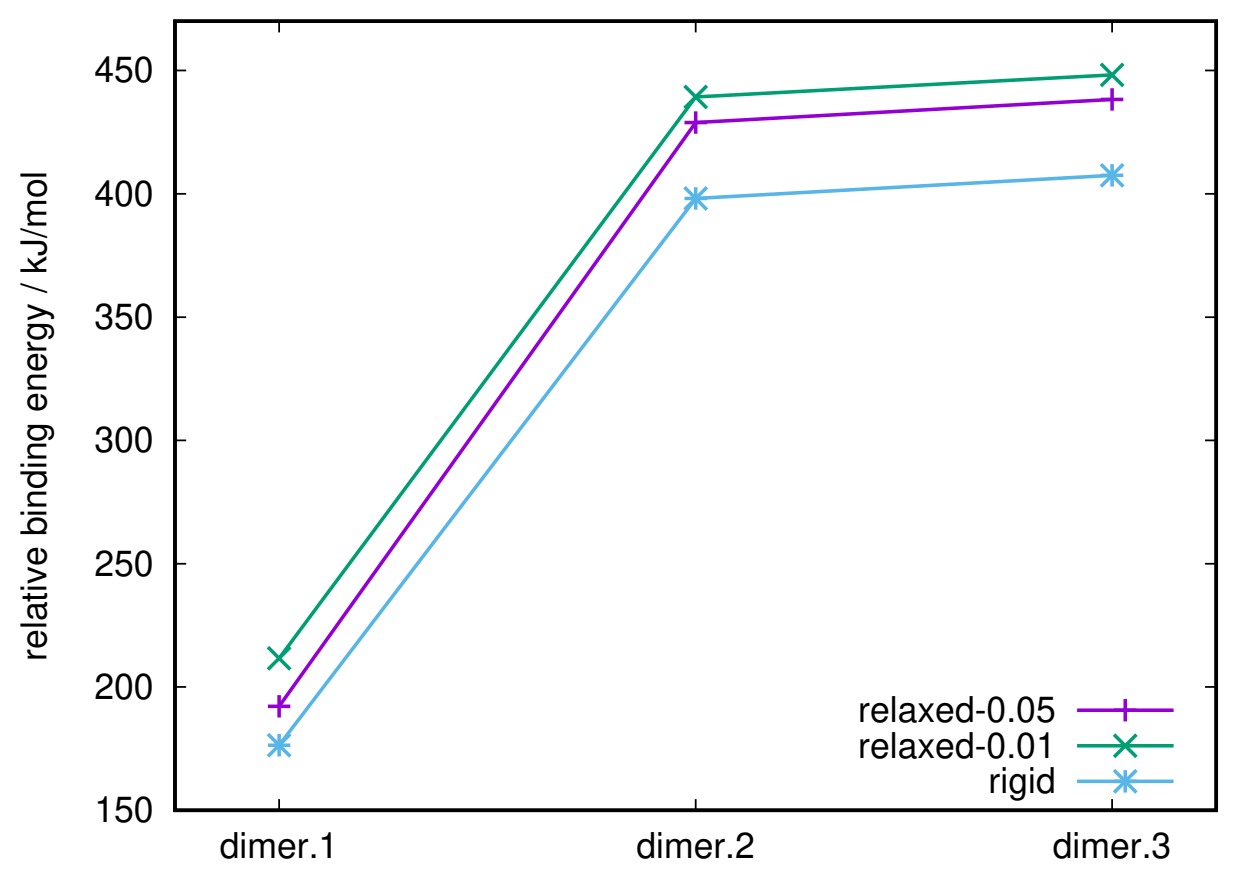

Figure S3: Total binding energy difference of three EP dimers on a $\mathrm{Pt}$ slab, relative to a fourth EP dimer binding energy, with the top $\mathrm{Pt}$ layer constrained with two different harmonic force constants $(0.05$ and 0.01 hartree/bohr ${ }^{2}$ ) and with a completely rigid $\mathrm{Pt}$ slab. Note that the ordinate does not start at zero $\mathrm{kJ} / \mathrm{mol}$, which would be the fourth $\mathrm{EP}$ dimer binding energy, to make the differences between these three data sets easier visible.

Note that the three lines show no tendency to cross, i.e., the energy ordering is maintained, and in fact they are almost parallel to each other. Additionally, the binding energy changes due to relaxing or not relaxing the top of the Pt slab are much smaller than the binding energy differences between these EP dimers.

This indicates that the approximation of using a fully rigid Pt slab not only saves computer time but also is physically reasonable and justified.

\section{Further quadrupole interactions of adsorbed EP molecules}

To further illustrate EP quadrupole interactions, as in Figs. 14 and 15 of the main paper, Fig. S4 shows yet another example from our best $(\mathrm{EP})_{8} @ \mathrm{Pt}(111)$ structures. Here, $z$-axis angles range from $20.9^{\circ}$ to $3.6^{\circ}$. Again, T-shaped and slipped-parallel configurations can be seen. In this case, one EP pair is almost in the most unfavorable 
aligned configuration, but the pair distance is fairly large and the quadrupoles are rather small.
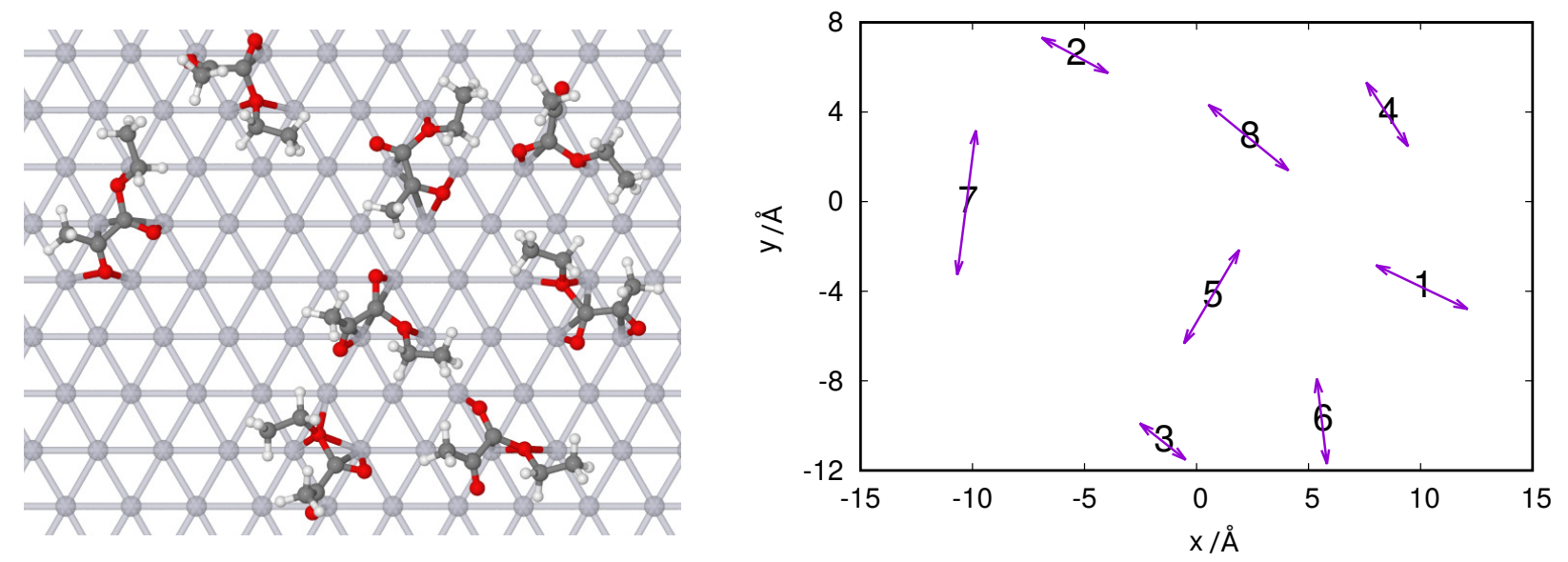

Figure S4: A third low-energy structure of 8 ethyl pyruvate molecules and its quadrupole pattern, displayed as in Figs. 14 and 15 of the main paper. T-shaped configurations are formed by the pairs 1-5 and 5-8, a slipped-parallel configuration by the pair $4-8$. Pair $2-8$ comes close to the unfavorable aligned configuration but is rather far apart and does not have a large quadrupole.

\section{References}

[1] W. Liu, V.G. Ruiz, G.-X. Zhang, B. Santra, X. Ren, M. Scheffler and A. Tkatchenko, "Structure and energetics of benzene adsorbed on transition-metal surfaces: densityfunctional theory with van der Waals interactions including collective substrate response", New J. Phys. 15 (2013) 053046.

[2] A. Freibert, J.M. Dieterich and B. Hartke, "Exploring self-organization of molecular tether molecules on a gold surface by global structure optimization", J. Comput. Chem. 40 (2019) 1978-1989.

[3] W. Chem, V. Madhavan, T. Jamneala and M.F. Crommie, "Scanning tunneling microscopy observation of an electronic superlattice at the surface of clean gold", Phys. Rev. Lett. 80 (1998) 1469.

[4] XTB documentation at https://xtb-docs.readthedocs.io/en/latest/xcontrol.html, accessed 11-Oct-2021. 